\title{
MONOCULTIVO DO CAMURIM, Centropomus undecimalis (BLOCH, 1792) EM VIVEIROS-REDE, PERNAMBUCO (BRAZIL).
}

Pereira, José Arlindo ${ }^{1,2}$ Santos, Gilvânia Alcântara Correia ${ }^{1}$

Macêdo, Sílvio José de $\mathrm{e}^{1,2}$ Santana, Marcio Francisco Alves de ${ }^{1}$ Departamento de Oceanografia/UFPE ${ }^{1}$ Bolsista do $\mathrm{CNPq}^{2}$

\section{RESUMO}

O presente trabalho foi desenvolvido na Base de Piscicultura de Itamaracá-PE, a partir de julho/92 com duração de 252 dias, tendo como objetivo fornecer subsídios sobre o monocultivo do camurim, Centropomus undecimalis. Os indivíduos foram estocados em três viveiros-rede de $9,00 \mathrm{~m}^{2}$ cada, instalados $\mathrm{em}$ um viveiro estuarino de $2,100 \mathrm{~m}^{2}$, em densidades de 3,$00 ; 6,00$ e 9,00 peixes/ $\mathrm{m}^{2}$, com pesos médios de $15,60 \mathrm{~g}, 16,60 \mathrm{~g}$ e $12,60 \mathrm{~g}$, respectivamente. A alimentação ministrada diariamente foi constituída basicamente de guaru vivo (Poecilia vivipara), a base de 7,00\% da biomassa existente em cada viveiro-rede nos três primeiros meses, $6,00 \%$ nos dois meses seguintes e 5,00 , a partir do sexto mês. As variáveis comprimento $(\mathrm{cm})$ e peso $(\mathrm{g})$ totais foram obtidas, mensalmente, através de amostras de $30,00 \%$ dos indivíduos existentes em cada viveiro-rede. Realizou-se semanalmente estudo das condições hidrológicas para determinação dos valores de oxigênio dissolvido, salinidade, temperatura, $\mathrm{pH}$ e transparência. Ao final do experimento constatou-se uma sobrevivência de $96,30 \%$ para os três viveiros-rede; um peso ganho/ind/dia variando de 0,39 a $0,65 \mathrm{~g}$; uma produção líquida/ha de $4.732,00$ a $8.517,60 \mathrm{~kg}$ e uma conversão alimentar de 6,20 a 7,10:1,00. A análise hidrológica apresentou mínimo de 2,92 $\mathrm{ml} / \mathrm{l}$ e um máximo de $7,51 \mathrm{ml} / 1$ para o oxigênio dissolvido; $23,57 \% 0$ e 40,37\% para a salinidade; $28,00^{\circ} \mathrm{C}$ e $31,30^{\circ} \mathrm{C}$ para a temperatura; 7,20 e 8,00 para $\mathrm{o} \mathrm{pH}$ e $35 \mathrm{~cm}$ e $98 \mathrm{~cm}$ para a transparência. Os resultados obtidos indicaram a viabilidade do monocultivo dessa espécie em viveiros-rede, sendo que a densidade de $9,00 \mathrm{ind} / \mathrm{m}^{2}$ foi a que apresentou a maior produtividade líquida/ha.

Palavras chave: Aquicultura, Centropomidae, viveiros-rede, Tropical

\section{ABSTRAT}

Monoculture of the common snook Centropomus undecimalis (Bloch, 1792) in Pond-net, Pernambuco (Brazil)

The main objective of this work was to supply subsidy about monocultive of the snook, Centropomus undecimalis. The work was carried out at Fishery station of Itamaracá-PE, from july / 92 to march / 93. Snooks with an average of $15,6,16,6$ and $12,6 \mathrm{~g}$ were raised in pond-nets of $9,00 \mathrm{~m}^{2}$ in densitys of $3,00,6,00$ and 9,00 fishes $/ \mathrm{m}^{2}$, respectively. The three pond-nets were instaled 
inside a estuarine pond of $2,100 \mathrm{~m}^{2}$. Snooks were feed daily with living lie bearing (Poecilia vivipara) on a basis of $7,00 \%$ of biomass at each pond-net during the three first months, $6,00 \%$ for the following two months and $5,00 \%$ there on. Monthly biometry (total lenght $(\mathrm{cm})$ and total weight $(\mathrm{g})$ ) were conducted through on $30,00 \%$ of the indiviuals present in each pond-net. Weekly, hydrologicals conditions (dissolved Oxygen, salinity, termperature, $\mathrm{pH}$ and water transparency) were monitored. Survival rate for the three pond-nets at the end of experiment ( 252 days) were similar: $96,38 \%$, while the rate of grouwth ( $g$ /ind/day) varying from 0,39 to 0,65 . Net production varied from $4,732,00$ to $8,517,60 \mathrm{~kg} / \mathrm{ha}$ and the food conversion rate varied from $6,20: 1$ to $7,10: 1$. Levels of dissolved oxigen varied from 2,92 to $7,51 \mathrm{ml} / \mathrm{l}$; sality varied from $23,57 \% 0$ to $40,37 \% 0$, temperature fluctuated from $28,0^{\circ} \mathrm{C}$ to $31,30^{\circ} \mathrm{C}, \mathrm{pH}$ ranged from 7,20 to 8,60 and transparency varied from 35,00 to $98,00 \mathrm{~cm}$. Results indicated that the monoculture of this species is viable and that $9,00 \mathrm{ind} / \mathrm{m}^{2}$ is density since it yelds the highest net produtivity /ha.

Key words: Aquacultura, Centropomidae, pond-net, Tropical

\section{INTRODUÇÃO}

Os camurins, como são denominados em nossa região, são peixes de ampla distribuição na costa brasileira e talvez os mais conhecidos e apreciados na nossa pesca desportiva e de subsistência.

São pertencentes a família Centropomidae e representados no nosso Estado pelas espécies Centropomus undecimalis (Bloch, 1972) e Centropomus parallelus Poey, 1960, com maior abundância para a primeira.

Possuem vasta distribuição geográfica abrangendo as Américas Tropical e Subtropical, além de leste da América Central e parte da América do Sul (Cervigon, 1966).

Habitam águas marinhas, salobras ou doces. Portanto podemos considerar que os estuários constituem o habitat principal desses peixes.

Além da natural adaptação a diferentes ambientes e salinidades, é um peixe místico, de hábitos gregários, que aceita bem o cativeiro.

Segundo Vasconcelos Filho et. al., (1980), o camorim é um peixe de regime alimentar eminentemente carnívoro, com a dieta constando principalmente de peixes e crustáceos, secundariamente de moluscos e outros animais, tendo o Centropomus undecimalis, demonstrado uma maior preferência por peixes, especialmente das familias gobiidae e gerreidae.

$\mathrm{Na}$ escolha de espécies para cultivo, deve-se levar em consideração vários atributos. Destacam-se entre estes atributos a rusticidade, a facilidade de manejo e a qualidade da carne, Pereira (1986).

O camurim, Centropomus undecimalis, é um peixe potencialmente apto para consumo, cultivado em viveiro nas áreas tropical e subtropical devido ao excelente sabor de sua carne e aceitável fonte de proteína de elevada quaglidade. 0 rendimento de $57,00 \%$ em sua filetagem é considerado elevado quando comparado ao de outras espécies comumente cultivadas, como o catfish de canal, Ictalurus punctatus.

Cerqueira (1989) avaliou as perspectivas para o cultivo de camurim em nosso país onde chama a atenção para a existência de múltiplas possibilidades, que vão desde o cultivo em viveiros estuarinos e aproveitamento das estruturas já existentes para peixe de água doce até o desenvolvimento de gaiolas ou cercos.

O cultivo de camurim em viveiros-rede apresenta facilidades de manejo, bem como da ocupação de menor espaço e ainda permite o cultivo paralelo de outros organismos, quando instalado em viveiro-suporte. Devido a sua rusticidade e resistência aos impactos ambientais, os centropomídeos adapta-se facilmente a tais ambientes, podendo serem cultivados em altas densidades.

Diferentemente da forma tradicional como são cultivados, servindo unicamente como controladores biológicos em cultivo principal, este trabalho pretende contribuir com uma forma mais propicia para a criação do camurim, estocados em diferentes densidades, ocupando um espaço reduzido e buscando obter uma maior produtividade.

\section{MATERIAL E MÉTODOS}

O presente experimento foi realizado na Base de Piscicultura do Departamento de Oceanografia da UFPE, localizada na ilha de Itamaracá $\left(7^{\circ} 34^{\prime}\right.$ $00^{\prime \prime}, 7^{\circ} 55^{\prime} 16^{\prime \prime} \mathrm{S}$ e $34^{\circ} 48^{\prime} 48^{\prime \prime}, 34^{\circ} 52^{\prime} 24^{\prime \prime} \mathrm{W}$ ), situada a $50 \mathrm{Km}$ ao norte de Recife.

Utilizou-se no presente estudo, três viveiros-rede (VR-1, VR-2 e VR-3) com $9,00 \mathrm{~m}^{2}$ de área $\times 1,00 \mathrm{~m}$ de altura, cada um; confeccionados com tela plástica (malha de $5,00 \mathrm{~mm}$ de nó a nó). Foram instalados em um viveiro estuarino de $2.100 \mathrm{~m}^{2}$ de profundidade de 0,80 a $1,00 \mathrm{~m}$. Estes viveiros-rede foram povoados com camurins jovens, Centropomus undecimalis, nas densidades de 3,$00 ; 6,00$ e 9,00 peixes $/ \mathrm{m}^{2}$ para o VR-1, VR-2 e VR-3, respectivamente.

O cultivo teve a duração de 252 dias, com início em 23/07/92. Os exemplares foram estocados com um comprimento que variou de 10,00 a 16,40 $\mathrm{cm}$ e peso médio de 12,60 a $16,60 \mathrm{~g}$, tendo sido capturados em charcos adjacentes aos viveiros com rede tipo mangote.

Mensalmente, a partir da data de povoamento, foram realizadas biometrias (comprimento e peso total) individuais em amostras de $30,00 \%$ dos peixes existentes em cada viveiro-rede.

De acordo com a biomassa previamente calculada após a biometria, era fornecida a alimentacão. Nos 3 (três) primeiros meses foram administrados 7,00\% da biomassa, nos 2 meses seguintes $6,00 \%$ e a partir do sexto mês de criação, $5,00 \%$ desta para cada viveiro-rede.

O alimento fornecido constava principalmente de guaru vivo, Poecilia vivipara, peixe pertencente a família poecilidae, podendo ocasionalmente ocorrer outros peixes de categoria inferior, como peixe-rei e mingula, dentre outros. $\mathrm{O}$

Trab. Oceanog. Univ. Fed. PE, Recife, 25:61-68, 1997 
alimento calculado para cada dia era oferecido 3(três) vezes na semana, pela manhã, em dias alternados.

Foi estabelecida uma estação de coleta de água no viveiro suporte, situada equidistante dos três viveiros-rede.

Semanalmente, nas primeiras horas da manhã, durante o período de cultivo, foram realizadas coletas de água superficial para a determinação do oxigenio dissolvido, salinidade e $\mathrm{pH}$. Simultaneamente eram observadas a profundidade, transparência e temperatura da água.

Os métodos de análise foram o de Winkler para o oxigênio e de MohrKnudsen para a salinidade, ambos modificado por Strickland e Parsons (1965), sendo que o $\mathrm{pH}$ obtido por intermédio de um $\mathrm{pH}$-meter 350 Methromag elétrico.

Para análise dos dados de produção liquida/ha $(\mathrm{kg})$, índice de crescimento específico, G (\%) e Incremento relativo em comprimento (RRI), utilizou-se o emprego das seguintes fórmulas:

Produção líquida/ha $(\mathrm{kg})=$

(biomassa final - biomassa inicial). 1000

área dos viveiros-rede em metros quadrado

$$
G(\%)=\frac{\ln W_{(T+\Delta T)}-\ln W_{T}}{T} \times 100 \quad R R I=\frac{L_{(T+\Delta T)}-L_{T}}{L_{T}}
$$

\section{RESULTADOS E DISCUSSÃO}

A Tabela I mostra os valores máximos, médios e mínimos dos principais fatores fisico-químicos no viveiro-suporte. A temperatura mais baixa $\left(28,00^{\circ} \mathrm{C}\right)$ foi registrada nos meses de novembro e dezembro/92, enquanto a mais alta $\left(31,30^{\circ} \mathrm{C}\right)$ foi detectada coincidentemente no primeiro mês citado. Essa variação de temperatura parece não influir sobre o desenvolvimento do camurim, porém cultivos efetuados por Okada et al. (1980) nos viveiros estuarinos de Itamaracá mostraram bons resultados, com mínima de $25,50^{\circ} \mathrm{C}$ e máxima de $30,50{ }^{\circ} \mathrm{C}$. Variações próximas também foram detectadas por Silva (1976), ou seja, mínima de $25,10^{\circ} \mathrm{C}$ (agosto) e máxima de $33,60{ }^{\circ} \mathrm{C}$ (dezembro).

A salinidade variou de $23,57 \% 0$ no mês de agosto/92 a $40,37 \% 0$ no mês de março/93. Silva (1967/1969) registrou salinidades bem mais acentuadas, da ordem de $54,00 \% 0$, durante as estações de verão em Pernambuco, em viveiros sem ou quase nenhuma renovação de água, não havendo, ao que parece, problemas imediatos para os camurins.

Quanto ao oxigênio dissolvido, a menor concentração $(2,92 \mathrm{ml} / \mathrm{l})$ foi verificada em janeiro/93, enquanto que a maior concentração $(7,51 \mathrm{~m} / \mathrm{l})$ foi observada em outubro/92. Maia et alo, (1980) detectaram um mínimo de 0,60 ml/ de oxigênio dissolvido em cultivo de camurim associado com curimã (Mugil liza) e tainha (Mugil curema) em viveiro natural. Este valor deve ter provocado uma limitação no desenvolvimento da biomassa confinada, tendo sido necessário uma circulação mecânica d'água no viveiro. Se levarmos em consideração que os viveiros-rede foram instalados em um viveiro-suporte, cuja porta d'água foi regulada para só permitir uma renovação a partir de uma maré de $1,90 \mathrm{~m}$, o valor mínimo aqui verificado situa-se dentro de um nível bastante aceitável. Sendo assim as variaçб̃es de oxigênio dissolvido no final do cultivo foram constatadas decrescentes. Até então, tais concentrações foram consideradas normais, portanto, segundo Eskinazi-Leça e Koening (1981) normalmente as águas desses viveiros não apresentam grandes variações em relação ao oxigênio dissolvido, estando as concentraç̃es entre $2,58 \mathrm{ml} / 1$ e $5,49 \mathrm{ml} / \mathrm{l}$. Cultivo de camurins em viveiros-rede realizado por Silva (1976), mostrou que o suprimento de oxigênio dissolvido foi suficiente, ou seja, mínimo de $3,10 \mathrm{ml} / 1 \mathrm{em}$ novembro e máximo de $5,48 \mathrm{ml} / 1$ em outubro.

Para o pH foram observadas variaçðes compreendidas entre 7,20 (agosto/92) e 8,60 (dezembro/92). O pH alcalino revelou ser um componente químico mais aceitável no experimento, resultados estes bastante aproximados dos obtidos por Okada et al., (1980); Maia et al., (1980) e Silva (1976).

A temperatura e a transparência oscilou conforme o período sazonal, enquanto a salinidade aumentou gradativamente com o tempo de cultivo.

No VR-1, os exemplares de Centropomus undecimalis, foram estocados com $15,60 \mathrm{~g}$ e despescados após 252 dias com $181,00 \mathrm{~g}$, o que correspondeu a um crescimento em peso de $0,66 /$ ind./dia. No VR-2 o incremento em peso foi de $0,53 \mathrm{~g} /$ ind./dia, uma vez que os indivíduos passaram de $16,60 \mathrm{~g}$ para $150,60 \mathrm{~g}$. Já no VR-3 o ganho de peso foi ainda menor, tendo sido povoado com exemplares de $12,60 \mathrm{~g}$ e no final do experimentos atingidos $111,30 \mathrm{~g}$.

Maia et al. (1980), cultivaram 265 curimãs com $78,30 \mathrm{~g}$ de peso médio em associação com 60 camurins com peso médio de $26,60 \mathrm{~g}$ num viveiro de 1200 $\mathrm{m}^{2}$, numa densidade total de 0,27 peixes $\mathrm{m}^{2}$, obtendo-se para os camurins um ganho de peso/ind./dia de $0,42 \mathrm{~g}$. Resultado este que se aproxima do verificado para o cultivo no viveiro-rede VR-3 deste trabalho, Obseva-se ainda, que aquele valor situa-se bem abaixo dos apresentados nos cultivos do VR-1 ( 3 peixes $/ \mathrm{m}^{2}$ ) - VR-2 (6 peixes $\left./ \mathrm{m}^{2}\right)$, com densidades bem mais elevadas do que a apresentada no policultivo entre curimãs e camurins.

Okada et al. (1980), efetuaram trabalho semelhante, em que fol povoado em mesma área 2,400 tainhas de $2,68 \mathrm{~g}$ e 120 eamurins com $2,05 \mathrm{~g}$ de pesos médios, com densidade total de 2,10 peixes $/ \mathrm{m}^{2}$. O ganho de peso obtido para os camurins foi de $0,324 \mathrm{~g} / \mathrm{ind} / \mathrm{dia}$, resultado este inferior, quando comparado aos obtidos neste trabalho.

Rocha e Okada (1980) obtiveram, para camurins, ganhos de peso/ind./dia de $0,43 \mathrm{~g}$ em cultivo associado com curimã, numa área de $1.200 \mathrm{~m}^{2}$ e densidade total de 0,16 peixes $/ \mathrm{m}^{2}$ e de $0,93 \mathrm{~g}$ em associação com tainha e curimã, em $8.000 \mathrm{~m}^{2}$ e densidade total $\mathrm{d}$ e 0,30 peixe $/ \mathrm{m}^{2}$. Verifica-se que o 
Tubela I - Valores mínimos, médios e máximos mensais da temperatura $\left({ }^{\circ} \mathrm{C}\right)$, transparência (m), coeficiente de extinção da huz, salinidade (\%०), pH, oxigênio dissolvido (mg.l-1)

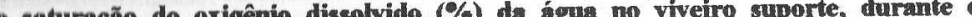
periodo de julho de 1992 a marco de 1993.

\begin{tabular}{l|l|l|l|l|l|l|l|l|l}
\hline & UUL & AGO & SET & OUT & NOV & DEZ & JAN & FEV & MAR \\
\hline \multirow{3}{*}{ TEMPERATURA } & 29,2 & 28,5 & 28,1 & 29,3 & 28,0 & 28,0 & 28,7 & 29,5 & 29,5 \\
& 29,5 & 29,0 & 29,0 & 30,2 & 30,5 & 28,7 & 29,1 & 29,8 & 30,1 \\
& 29,8 & 29,3 & 30,1 & 30,5 & 31,3 & 29,0 & 29,5 & 30,0 & 30,8 \\
\hline \multirow{3}{*}{ TRANSPAREANCIA } & 0,70 & 0,65 & 0,63 & 0,47 & 0,72 & 0,52 & 0,54 & 0,48 & 0,35 \\
& 0,84 & 0,85 & 0,75 & 0,73 & 0,77 & 0,62 & 0,59 & 0,56 & 0,48 \\
& 0,98 & 0,98 & 0,82 & 0,82 & 0,81 & 0,75 & 0,64 & 0,66 & 0,60 \\
\hline \multirow{3}{*}{ OOEF. EXI. LUZ } & 1,73 & 1,73 & 2,07 & 2,07 & 2,10 & 2,27 & 2,66 & 2,57 & 2,83 \\
& 2,02 & 2,00 & 2,27 & 2,32 & 2,22 & 2,74 & 2,88 & 3,01 & 3,56 \\
& 2,43 & 2,61 & 2,7 & 3,62 & 2,36 & 3,27 & 3,15 & 3,54 & 4,86 \\
\hline \multirow{5}{*}{ SALINIDADE } & 24,39 & 23,57 & 24,33 & 26,25 & 29,07 & 29,34 & 34,75 & 36,36 & 36,36 \\
& 24,66 & 25,01 & 25,25 & 28,72 & 31,02 & 32,48 & 35,48 & 37,36 & 38,27 \\
& 24,93 & 26,15 & 26,01 & 31,16 & 32,57 & 35,56 & 36,09 & 38,50 & 40,37 \\
\hline \multirow{5}{*}{ pH } & 7,74 & 7,20 & 7,70 & 7,72 & 7,22 & 8,29 & 8,24 & 8,42 & 7,99 \\
& 7,91 & 7,61 & 8,00 & 8,01 & 8,03 & 8,47 & 8,31 & 8,47 & 8,11 \\
& 8,09 & 8,06 & 8,20 & 8,35 & 8,30 & 8,60 & 8,40 & 8,51 & 8,18 \\
\hline \multirow{3}{*}{ OXIG. DISSOL. } & 4,38 & 5,64 & 4,70 & 4,69 & 4,79 & 4,78 & 2,92 & 3,90 & 3,03 \\
& 5,03 & 5,97 & 6,13 & 6,04 & 5,65 & 5,03 & 4,01 & 4,35 & 3,76 \\
& 5,69 & 6,26 & 7,20 & 7,51 & 6,89 & 5,20 & 4,46 & 5,74 & 4,44 \\
\hline \multirow{2}{*}{ SAT. OXIG.DISS } & 94,80 & 120,00 & 102,62 & 101,51 & 103,90 & 105,52 & 66,36 & 90,28 & 72,14 \\
& 108,40 & 127,84 & 131,26 & 134,52 & 128,12 & 111,28 & 90,72 & 100,93 & 88,06 \\
& 122,10 & 134,33 & 152,54 & 144,54 & 158,75 & 116,07 & 100,68 & 132,56 & 103,74 \\
\hline
\end{tabular}

Tabela II - Informaç̃es gerais sobre a criação intensiva do camorim Centropomus undecimalis (Bloch, 1792), durante o período de cultivo.

\begin{tabular}{|c|c|c|c|}
\hline VIVEIRO-REDE $\left(9 \mathrm{~m}^{2}\right)$ & VR-1 & VR-2 & VR-3 \\
\hline DATA DE ESTOCAGEM & $23 / 07 / 92$ & $23 / 07 / 92$ & $23 / 07 / 92$ \\
\hline TAXA DE ESTOCAGEM (ind. $\mathrm{m}^{-2}$ ) & 3 & 6 & 9 \\
\hline DENSIDADE VIVEIRO-REDE & 27 & 54 & 81 \\
\hline DIAS DE EXPERIMENTO & 251 & 251 & 251 \\
\hline PESO MÉDIO INICIAL (g) & 15,55 & 16,66 & 12,59 \\
\hline PESO MÉDIO FINAL (kg) & 181,00 & 150,64 & 111,29 \\
\hline COMPRIMENTO MÉDIO INICIAL $(\mathrm{cm})$ & 13,15 & 13,16 & 12,38 \\
\hline COMPRIMENTO MÉDIO FINAL $(\mathrm{cm})$ & 30,51 & 27,62 & 24,91 \\
\hline BIOMASSA INICIAL (g) & 420,00 & 900,00 & 1020,00 \\
\hline BIOMASSA FINAL (g) & 4706,00 & 7833,28 & 8680,62 \\
\hline BIOMASSA GANHA (g) & 4286,00 & 6933,28 & 7660,62 \\
\hline SOBREVIVÊNCIA/N ${ }^{\circ}$ ind. & $26=$ & 52 & 78 \\
\hline FINAL $/ \%$ & 96,30 & 96,30 & 96,30 \\
\hline PESO GANHO/DIA (g) & 0,66 & 0,53 & 0,39 \\
\hline BIOMASSA GANHA/HÁDIA (g) & $18.972,99$ & $30.691,81$ & $33.911,55$ \\
\hline PRODUÇÃO LÍQUIDA (kg) & 4762,22 & 7703,64 & 8511,79 \\
\hline CONV ALIM. APAR. (kg) & $6,6: 1$ & $7,1: 1$ & $6,2: 1$ \\
\hline INCREMENTO DA PRODUÇÃO (g/dia) & 17,07 & 27,62 & 30,52 \\
\hline ALIMENTO FORNECIDO $(\mathrm{g})$ & 28,30 & 49,49 & 47,31 \\
\hline INDICE DE CRESC. ESPEC. (\%) & 0,98 & 0,88 & 0,87 \\
\hline
\end{tabular}

primeiro valor obtido foi superior apenas ao verificado no VR-3 $\left(9\right.$ peixes $\left./ \mathrm{m}^{2}\right)$; já o segundo valor apresentado esteve acima dos observados no presente experimento. Se levarmos em consideração as densidades de estocagem num e noutro experimentos, verifica-se novamente as vantagens no monocultivo, com taxas elevadas em viveiros-rede.

Silva (1992), cultivando camurins em viveiros de água doce como controlador biológico para tilápia, em 3 (três) viveiros com densidade total de 0,80 peixes $/ \mathrm{m}^{2}$, obteve ganhos de peso/ind /dia de 0,$81 ; 0,58$ e $0,33 \mathrm{~g}$; resultados próximos aos encontrados nesse trabalho. Os valores referentes ao crescimento, produção, alimento fornecido; assim como o coeficiente de conversão alimentar aparente e taxa de sobrevivência encontram-se na Tabela II.

\section{CONCLUSÕES}

De acordo com os objetivos propostos para este trabalho, concluímos para Centropomus undecimalis, embasado nos resultados obtidos que:

- Cultivado em viveiros-rede fixos com dieta viva, demonstrou boa adaptabilidade a este sistema de cultivo, mantendo-se dentro dos padôes normais de crescimento;

- O monocultivo dessa espécie em viveiros-rede é viável, sendo a densidade de $9,00 \mathrm{ind} / \mathrm{m}^{2}$ a de maior produtividade líquida/ha

\section{REFERÊNCIAS BIBLIOGRÁFICAS}

CERQUEIRA, V. R. Análise e perspectiva para o cultivo do robalo Centropomus sp, no litoral brasileiro. Florianópolis, 1989. 15p. Tese (Professor Assistente em Piscicultura) - Universidade Federal de Santa Catarina.

CERVIGON, F. M. Los peces marinos de Venezuela. Caracas: Fundación La Salle de Ciencias Naturales, 1966. 438p.

ESKINAZI-LEÇA, E.; KOENING, M. L. Estudo ecológico da Região de Itamaracá - Pernambuco, Brasil. XII. Fitoplâncton dos viveiros estuarinos. In: CONGRESSO BRASILEIRO DE ENGENHARIA DE PESCA, 2, 1981, Recife. Anais...Recife: Associação dos Engenheiros de Pesca do Estado de Pernambuco, 1981. P. 221-232.

MAIA, E. P.; ROCHA, I. P.; OKADA, Y. Cultivo arraçoado de curimã (Mugil brasiliensis Agassiz, 1829) em associação com tainha (Mugil curema Valenciennes, 1836) e camurim (Centropomus undecimalis (Bloch, 1792)), em viveiros estuarinos de Itamaracá-PE. In: SIMPÓSIO BRASILEIRO DE AQUICULTURA, 1, 1978, Recife. Anais...Rio de Janeiro: Academia Brasileira de Ciências, 1980. p.141-149.

OKADA, Y.; MAIA, E. P.; ROCHA, I. P. Cultivo arraçoado de tainha (Mugil curema Valenciennes, 1836) em associação com camurim (Centropomus undecimalis (Bloch, 1792)) e carapeba (Eugerres brasilianus, 1830) em viveiros estuarinos de Itamaracá-PE. In: SIMPÓSIO BRASILEIRO DE AQUICULTURA, 1, 1978, Recife. Anais...Rio de Janeiro: Academia Brasileira de Ciências, 1980. p.131-139.

Trab. Oceanog. Univ. Fed. PE, Recife, 25:61-68, 1997 
PEREIRA, J. A Cultivo monosexo de machos de Oreochromis niloticus (Linnaeus, 1757) e machos híbridos de O. honorum (Trewavas, 1966) (machos) $\mathrm{x} O$. niloticus (fềmeas), em sistema intensivo. Aspectos quantitativos. (Pisces, Osteichthyes, Cichlidae). São Carlos, 1986. 99p. Tese (Doutorado em Ecologia e Recursos Naturais) - Universidade Federal de São Carlos, 1986.

PEREIRA, J. A.; OKADA, Y. Experimentos de policultivo entre curimã (Mugil brasiliensis Agassiz, 1829) e camurim (Centropomus undecimalis (Bloch, 1792)) em viveiros estuarinos (Itamaracá-Pernambuco). In: SIMPÓSIO BRASILEIRO DE AQUICULTURA, 1, 1978, Recife. Anais...Rio de Janeiro: Academia Brasileira de Ciências, 1980. p.163-173.

SILVA, J. E. Fisiologia do camorim, Centropomus undecimalis (Bloch, 1792). Estudo experimental do crescimento em ambiente confinado. São Paulo, 1976. 101p. Tese (Doutorado em Ciências) - Universidade de São Paulo.

SILVA, A. L. N. Efeito da predação do camorim Centropomus undecimalis (Bloch, 1792) - PISCES: Centropomidae - sobre a tilápia Oreochromis niloticus (Linnaeus, 1758) cultivados em viveiros de água doce. Florianópolis, 1992. 105p. Dissertação (Mestrado em Aquicultura). Universidade Federal de Santa Catarina.

SILVA, A. L. N.; LANDAU, M. F.; FAULKNER, B. E. Culinary value and composition of wild and captive common snook Centropomus undecimalis. Flor. Sci. Fort Pierce, 48(4):193-196, 1985.

STRICKLAND, J. D. H.; PARSONS, T. R. A manual of sea water analysis. Bull. Fish. Res. Bd. Can., 125:1-205, 1972.

VASCONCELOS FILHO, A. L.; AZEVEDO, S. B.; ALVES, M.L. C. Regime alimentar dos camorins Centropomus undecimalis (Bloch, 1792) e Centropomus parallelus (Poey, 1860) do Canal de Santa Cruz (PernambucoBrasil). In: SIMPÓSIO BRASILEIRO DE AQUICULTURA, 1, 1978, Recife. Anais...Rio de Janeiro: Academia Brasileira de Ciências, 1980. p.175-184. 